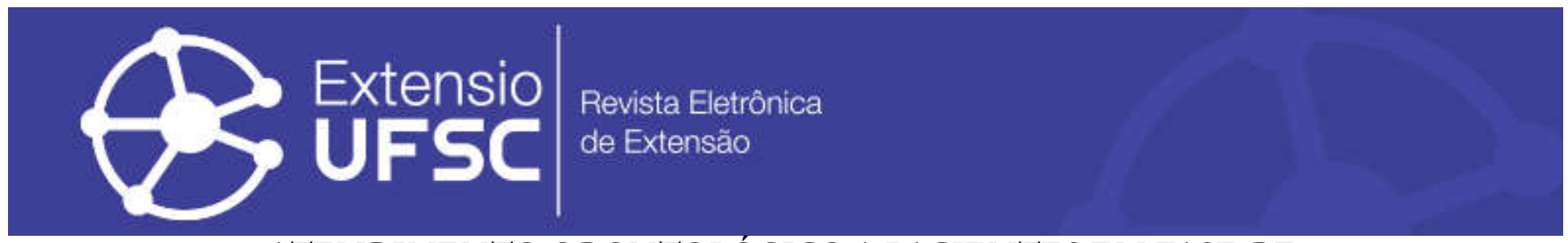

\title{
ATENDIMENTO ODONTOLÓGICO A PACIENTES EM FASE DE PRÉ-TRANSPLANTE HEPÁTICO: PROPOSTA DE PROTOCOLO
}

\author{
Sandro Felipe dos Santos Faria \\ Universidade Federal de Minas Gerais \\ fariasandro@outlook.com \\ Lia Silva de Castilho \\ Universidade Federal de Minas Gerais \\ liasilvacastilho@gmail.com
}

\author{
Agnaldo Soares Lima \\ Universidade Federal de Minas Gerais \\ agnaldo@gold.com.br \\ Rafael Paschoal Esteves Lima \\ Universidade Federal de Minas Gerais \\ rafaelpaschoalesteves@yahoo.com.br
}

Maria Elisa Souza e Silva Universidade Federal de Minas Gerais mariaelisa1956@gmail.com

\section{Resumo}

O número de pacientes que aguardam o transplante hepático tem aumentado mundialmente. Sua avaliação e tratamento odontológicos são recomendados antes do transplante hepático, com o objetivo de eliminar focos de infecções bucais, evitando complicações orais e infecções generalizadas após a terapia imunossupressora.Para isso, uma padronização de condutas clínicas odontológicas deve ser empregada para uniformização dos tipos de tratamento para determinados diagnósticos. O objetivo deste estudo é propor um protocolo de atendimento odontológico a pacientes candidatos a transplante hepático. Os procedimentos odontológicos realizados no projeto de extensão "Assistência Odontológica a Pacientes Transplantados de Fígado" demonstraram que podem ser realizados com segurança, desde que as devidas alterações da condição sistêmica sejam avaliadas, observando exames como coagulograma e realizando a escolha da correta medicação e trabalhando em conjunto com a equipe multidisciplinar irão garantir uma melhor qualidade de vida para os pacientes.

Palavras-chave: Transplante de Fígado. Assistência Odontológica. Saúde Bucal.

\section{DENTAL CARE FOR PATIENTS UNDERGOING PRE-LIVER TRANSPLANTATION: PROTOCOL PROPOSAL}

Abstract

The number of patients awaiting liver transplantation has increased worldwide. Its dental evaluation and treatment are recommended before liver transplantation, with the objective of eliminating foci of oral infections, avoiding oral complications and generalized infections after immunosuppressive therapy. For this, a standardization of dental clinical procedures must be used to standardize the types of treatment for certain diagnoses. The aim of this study is to propose a dental care protocol for patients who are candidates for liver transplantation. The dental procedures performed in the extension project "Dental Assistance to Liver Transplanted Patients", demonstrated that they can be performed safely, as long as the appropriate changes in the systemic condition are evaluated, observing exams such as coagulogram and making the choice of the correct medication and working together with the multidisciplinary team will ensure a better quality of life for patients.

Key words: Liver Transplantation. Dental Care. Oral Health.

\section{ATENCIÓN DENTAL PARA PACIENTES SOMETIDOS A TRASPLANTEPREVIO AL HÍGADO: PROPUESTA DE PROTOCOLO}

\section{Resumen}

El número de pacientes que esperan un trasplante de hígado ha aumentado en todo el mundo. Se recomienda evaluación y tratamiento odontológico antes del trasplante de hígado, con el fin de eliminar focos de infecciones bucales, evitando complicaciones bucales e infecciones generalizadas tras la terapia inmunosupresora. Para ello, se debe utilizar una estandarización de los procedimientos clínicos dentales para estandarizar los tipos de tratamiento para ciertos diagnósticos. El objetivo de este estudio es proponer un protocolo de atención odontológica para pacientes candidatos a trasplante hepático. Los procedimientos dentales realizados en el proyecto de extensión "Asistencia Dental a Pacientes Trasplantados de Hígado", demostraron que se pueden realizar de manera segura, siempre y cuando se evalúen los cambios adecuados en la condición sistémica, observando exámenes como el coagulograma y haciendo la elección de la medicación correcta y funcionando junto con el equipo multidisciplinar aseguraremos una mejor calidad de vida a los pacientes.

Palabras clave: Trasplante de Hígado. Asistencia Dental. Salud Bucal. 
Atendimento odontológico a pacientes em fase de pré-transplante hepático: proposta de protocolo

\section{INTRODUÇÃO}

O fígado é um órgão vital para a vida, pois participa da síntese e metabolismo de proteínas, de colesterol e bile, produz fatores de coagulação e metaboliza medicamentos, desintoxica metabólitos e armazena vitamina K (PERDIGÃO et al., 2012; LEVENSON e KEENAN, 2013). Tem papel importante na hemostasia e sua função deve ser avaliada antes de qualquer procedimento invasivo (WARD e WEIDEMAN, 2006; HELENIUSHIETALA et al., 2012; SILVA-SANTOS et al., 2012; RADMAND et al., 2013).

Várias são as doenças que podem comprometer as funções hepáticas, como hepatites, doenças de depósito, abuso de álcool, doenças hereditárias e genéticas que levam à cirrose, resultado de um processo crônico de destruição das células hepáticas. A cirrose ocorre de maneira difusa, com formação de cicatrizes e nódulos, sendo considerada um estágio terminal na evolução patológica do fígado (Di PROFIO et al., 2017; GBD, 2020).

O transplante é uma conduta terapêutica indicada para pacientes com doenças crônicas ou incuráveis do fígado. Consiste na cirurgia de substituição do órgão doente por um fígado saudável, doado de um paciente diagnosticado com morte encefálica (KIMURA et al., 2020) ou, menos frequentemente, realizado com parte do fígado de um doador vivo.Por ano, mais de 6.000 procedimentos de transplante hepático são realizados nos Estados Unidos(NATIONAL DATA, 2020). No Brasil, o número de doações chegou a 13,2 por milhão de habitantes. O governo federal brasileiro de 2013 a 2015 tem aumentado o número total e promovido a descentralização das equipes de transplante em todo o país (MEIRELLES et al., 2015).

A avaliação odontológica é um pré-requisito recomendado, tendo em vista que a terapia imunossupressora induzida por drogas, necessária para o sucesso de transplante de órgãos, traz consigo o risco de infecções oportunistas. Desta forma, focos de infecções dentais devem ser eliminados antes do transplante, com o objetivo de evitar infecções generalizadas de origem bucal (PERDIGÃO et al., 2012; HELENIUS-HIETALA et al., 2012; SILVA-SANTOS et al., 2012; LEVENSON e KEENAN, 2013; DIDILESCU et al., 2020). Mais de $90 \%$ dos pacientes portadores de doença hepática crônica apresentam alterações na coagulação, o que sugere aumento do risco de hemorragia (SILVA SANTOS et al., 2012).Procedimentos cirúrgicos são atrasados ou cancelados pelo temor de sangramento (RADMAND et al., 2013). Por isso, os cirurgiões-dentistas devem conhecer as particularidades dos pacientes com lesões hepáticas, candidatos ao transplante, para que 
Atendimento odontológico a pacientes em fase de pré-transplante hepático: proposta de protocolo

possam realizar um tratamento odontológico com segurança (HELENIUS-HIETALA et al., 2012; SILVA SANTOS et al., 2012; RADMAND et al., 2013).

Os objetivos do presente estudo são a apresentação de protocolo de atendimento odontológico a pacientes candidatos a transplante hepático, considerando a evidência científica atual, e revisar a literatura atual acerca dos cuidados ao paciente com doença hepática crônica em fase pré-transplante durante o atendimento odontológico. Este protocolo foi originado de uma atividade de pesquisa realizada por alunos do Projeto de Extensão "Assistência Odontológica a Pacientes Transplantados de Fígado", da Faculdade de Odontologia da UFMG.

\section{METODOLOGIA}

Foi realizada pesquisa bibliográfica por estudante do projeto de extensão "Assistência Odontológica a Pacientes Transplantados de Fígado" nas bases de dados Pubmed e Lilacs, utilizando-se as seguintes palavras-chave: Liver Transplantation, Oral Health, Dental Care e Chronic Liver Disease. Não houve restrição de idioma e a pesquisa foi limitada a artigos publicados a partir do ano 2005. Os artigos foram selecionados através da leitura do título, do resumo e/ou do texto completo considerando sua relevância e sua adequação aos objetivos do presente trabalho. Para tanto, deveriam conter informações sobre exames clínicos como contagem de plaquetas, avaliação da hemoglobina/hematócrito, Tempo de Protrombina (PT), a razão normalizada internacional (RNI) e Tempo de Tromboplastina Parcial (PT'T) e suas relações com o tratamento odontológico invasivo ou não. O uso de medicamentos em odontologia e seus possíveis efeitos colaterais e reações adversas ao medicamento mais frequentes entre pacientes com hepatopatias também foram levantados. Finalmente, foram levantadas as técnicas locais e sistêmicas indicadas para o controle do sangramento durante a realização de procedimentos invasivos nestes pacientes.

\section{REVISÃO DA LITERATURA}

\section{A doença hepática crônica e o transplante de fígado}

As cirroses secundárias às hepatites $\mathrm{B}$ e $\mathrm{C}$, a cirrose alcoólica e a cirrose pós esteatohepatite não-alcóolica (non-alcoholic steatohepatitis, NASH) são as condições mais 
Atendimento odontológico a pacientes em fase de pré-transplante hepático: proposta de protocolo

frequentemente associadas à indicação de transplante em adultos. Em crianças, a indicação mais comum para o transplante é a atresia biliar (GBD, 2020).

Também existem contraindicações ao transplante hepático. Fatores desfavoráveis para a indicação à cirurgia são alterações anatômicas como tromboses venosas do sistema porta, presença de neoplasia, a falência não corrigível de outro órgão ea reduzida probabilidade de sobrevivência em longo prazo ou baixa qualidade de vida após o transplante (FOX, 2014). Adicionalmente, as infecções de origem bucal têm sido consideradas causa para o cancelamento ou adiamento da cirurgia de transplantedo orgão (HELENIUS-HIETALA et al., 2012).No Brasil, a alocação de fígado do doador é baseada no sistema MELD, do inglês Model for End-stage Liver Disease, desde 2006 (FREITAS et al., 2019). Neste modelo matemático, dados de exames laboratoriais e também algumas situações especiais determinam a pontuação de gravidade do paciente, que ordena a lista de candidatos ao transplante.

As infecções são um motivo de preocupação após o transplante hepático (SILVA SANTOS et al., 2012). Mais da metade dos receptores apresentam complicações infecciosas no primeiro ano após o procedimento, e cerca de um terço apresenta infecções graves nos anos subsequentes ( $\AA B$ ERG et al., 2014).

A incidência de infecções pós-transplante é significativamente maior entre indivíduos não submetidos ao tratamento odontológico prévio ao transplante (HELENIUS-HIERALA et al., 2012). Pacientes com doenças hepáticas possuem maior prevalência de petéquias, gengivite, sangramento gengival, candidíase, queilite, xerostomia e periodontite (SILVA SANTOS et al., 2012) e um grande número de dentes extraídos e lesões cariosas (LINS et al., 2017). A condição bucal insatisfatória tem sido associada à elevação da pontuação do escore MELD (ÅBERG et al., 2014).

A trombocitopenia, na qual o número de plaquetas atinge valores inferiores a $150.000 / \mathrm{mm}^{3}$, é uma complicação encontrada em indivíduos com hipertensão portal secundária a doenças crônicas do fígado ou cirrose, cujas causas incluem sequestro esplênico de plaquetas, supressão da produção de plaquetas na medula óssea e diminuição da atividade do fator de crescimento hematopoiético trombopoetina. A trombocitopenia leve, caracterizada pela contagem de plaquetas entre $75.000 / \mathrm{mm}^{3}$ e $150.000 / \mathrm{mm}^{3}$, não apresenta significado clínico e, portanto, não interfere no tratamento odontológico ou no gerenciamento de decisões. A trombocitopenia moderada, com níveis de plaquetas entre $50.000 / \mathrm{mm}^{3} \mathrm{e} 75.000 / \mathrm{mm}^{3}$, é observada em aproximadamente $13 \%$ dos pacientes 
Atendimento odontológico a pacientes em fase de pré-transplante hepático: proposta de protocolo

cirróticos. Quando a contagem de plaquetas atinge níveis inferiores a 50.000/ $\mathrm{mm}^{3}$, a trombocitopenia é considerada grave e pode ser associada com morbidade significativa (AFDHAL et al., 2008).

\section{Terapêutica medicamentosa}

Em indivíduos com doença hepática devem ser consideradas alterações na dosagem ou nos intervalos de administração de analgésicos, pois as doenças hepáticas podem alterar o metabolismo das drogas (CRUZ PAMPLONA et al., 2011). O uso agudo ou crônico de paracetamol em doses máximas de $2 \mathrm{~g}$ ao dia, em indivíduos com hepatopatias, é considerado seguro (RADMAND et al., 2013).A dipirona na dosagem de 500mg TID no período de até 72 horas é também uma opção aceitável nesse particular grupo de indivíduos(ZAPATER et al., 2015).Entretanto, o uso de aspirina é contraindicado em indivíduos com alterações na hemostasia (CRUZ PAMPLONA et al., 2011).Analgésicos narcóticos devem ser evitados devido ao risco de encefalopatia hepática (SILVA SANTOS et al., 2012).O uso de fármacos anti-inflamatórios não esteroídes (AINES), devido ao potencial para sangramento gastrointestinal e complicações renais em indivíduos com cirrose, está contraindicado (RADMAND et al., 2013). Ansiolíticos, em indivíduos com doença hepática, devem ser usados com cautela, pois o tempo de ação dessa classe pode ser prolongado (SILVA SANTOS et al., 2012).

Quando a disfunção hepática estiver associada a alterações imunológicas,como nas doenças autoimunes, a profilaxia antibiótica está indicada para procedimentos invasivos(CRUZ PAMPLONA et al., 2011). A profilaxia antibiótica deve ser recomendada considerando a extensão do procedimento e a gravidade da doença hepática. Amoxicilina, ácido clavulânico ou cefalosporinas são indicados (RADMAND et al., 2013). A clindamicina apresenta seu metabolismo prolongado em indivíduos com doenças hepáticas e, portanto, poderia contribuir com a degeneração do fígado (CRUZ PAMPLONA et al., 2011).Entretanto, ainda não existem diretrizes padrão para o uso de profilaxia antibiótica em pacientes pré-transplante hepático.A decisão sobre a realização ou não da profilaxia antibiótica deve ser estabelecida junto ao médico responsável.

\section{Procedimentos invasivos}

Devido ao risco hemorrágico, a avaliação pré-operatória através de exames complementares do paciente é obrigatória, independentemente do tipo de procedimento 
Atendimento odontológico a pacientes em fase de pré-transplante hepático: proposta de protocolo

odontológico que será realizado. A propedêutica deve incluir um hemograma completo, para contagem de plaquetas e avaliação da hemoglobina/hematócrito. Tempo de Protrombina (PT), RNI e Tempo de Tromboplastina Parcial (PTT) também devem ser solicitados (CRUZ PAMPLONA et al., 2011) embora haja clara limitação destes exames para prognosticar sangramento em hepatopatas.Caso haja necessidade de realização de procedimentos cirúrgicos, deve ser realizada uma técnica cuidadosa, compressão da região para evitar sangramentos e utilização de agentes hemostáticos locais como celulose regenerada oxidadae ácido tranexâmico, e agentes sistêmicos como plasma, plaquetas e vitamina $\mathrm{K}$, se necessário. Qualquer procedimento odontológico invasivo pode ser realizado com nível de plaquetas igual ou superior a $50.000 / \mathrm{mm}^{3}$. A transfusão préoperatória dos produtos derivados do sangue pode ser necessária com base na extensão do procedimento odontológico e as alterações de coagulação presentes. Após a transfusão de produtos derivados do sangue, o procedimento deve ser rapidamente realizado devido à degradação dos fatores de coagulação e sequestro de plaquetas. A determinação da contagem de plaquetas e/ou PT/RNI pode ser feita,se o tempo para sua obtenção não comprometer a eficiência da transfusão realizada. Para obtenção da hemostasia, recomenda-se ainda o uso de agentes hemostáticos locais como trombina bovina tópica, microfibras de colágeno ou ácido aminocapróico (AFDHAL et al., 2008; SILVA SANTOS et al., 2012; RADMAND et al., 2013).

A literatura diverge em relação aos resultados de contagem de plaquetas e/ou RNI em relação ao índice de complicações pós-operatórias. A maioria das exodontias pode ser realizadas sem complicações hemorrágicas. Maior incidência de sangramento é observada em indivíduos com trombocitopenia grave $\left(<50.000 / \mathrm{mm}^{3}\right)$ ou em indivíduos que apresentavam falha na síntese de fatores de coagulação (RNI> 1,5) (HELENIUSHIETALA et al., 2012). Encontrou-se baixo risco de sangramento após exodontias em indivíduos com $\mathrm{RNI} \leq 2,50$ e contagem de plaquetas $\geq 30.000 / \mathrm{mm}^{3}$, sem a necessidade de transfusões de sanguedes de que sejam realizados em ambiente hospitalar(PERDIGÃO et al., 2012).Outros autores, entretanto, recomendam o RNI máximo de 3,5 para a realização de procedimentos invasivos (AFDHAL et al., 2008; SILVA SANTOS et al., 2012).

As técnicas locais indicadas para o controle do sangramento são o concentrado de fibrinogênio humano, a celulose oxidada, o ácido tranexâmico, o spray de cianoacrilato, a anestesia intraligamentar, o ácido aminocapróico, a trombina em pó, a cola de fibrina e a compressão do alvéolo. Para a terapia sistêmica, podem ser necessáriasinfusões de 
Atendimento odontológico a pacientes em fase de pré-transplante hepático: proposta de protocolo

produtos do sangue (FFP, plaquetas, concentrado de hemácias, crioprecipitado, fator VIIa recombinante), fluidos intravenosos, ácido tranexâmico e injeções de vitamina K(WARD e WIEDEMAN, 2006), mas a indicação desta terapêutica deve ser baseada em avaliações hematológicas.

A utilização de anestésico local associado a vasoconstritor do tipo epinefrina pode contribuir para a melhora da hemostasia e tem demonstrado ser segura, desde que a dose não exceda $7 \mathrm{mg} / \mathrm{kg}($ CRUZ PAMPLOLNA et al., 2011).

\section{DISCUSSÃO}

Problemas pré-existentes na boca podem evoluir para um processo agudo e aumentar a possibilidade de complicações, trazendo risco à saúde e à qualidade de vida dos pacientes que demandam transplante de fígado. $\mathrm{O}$ tratamento odontológico em pacientes candidatos a transplante tem um papel fundamental no conjunto de medidas que visam evitar que infecções de origem bucal possam se transformar em infecções generalizadas (PERDIGÃO et al., 2012; SILVA SANTOS et al., 2012; LEVENSON e KEENAN, 2013; RADMAND et al., 2013). Essas contribuem para uma estadia mais prolongada no hospital, gerando mais custos, e maior risco de insucesso do transplante.

O atendimento odontológico pré-transplante garante melhor qualidade de vida póstransplante aos pacientes (RADMAND et al., 2013). Com isso, o cirurgião-dentista como parte da equipe que realiza o atendimento multidisciplinar, tem um papel importante para o sucesso do transplante.

O tratamento odontológico de indivíduos com hepatopatias requer a realização da coleta de toda a história clínica, identificação minuciosa dos medicamentos em uso e verificação ou solicitação de exames laboratoriais para avaliação do hemograma e do coagulograma. Em seguida, deve ser feito um cuidadoso exame extra e intraoral (CRUZ PAMPLONA et al., 2011; SILVA SANTOS et al., 2012). Assim, o cirurgião-dentista irá determinar quais os cuidados ou procedimentos serão adotados para que o tratamento planejado possa ser adequadamente executado. Esses cuidados irão garantir preparação correta e monitoramento das doenças bucais antes do transplante.

O fígado é fundamental no metabolismo de drogas e os pacientes com doença hepática podem apresentar capacidade diminuída de transformação e eliminação das mesmas. Assim, caso haja necessidade de prescrição de medicamentos, deve-se analisar 
Atendimento odontológico a pacientes em fase de pré-transplante hepático: proposta de protocolo

quais classes, intervalos e dosagens são seguros para esse grupo de pacientes (CRUZ PAMPLONA et al., 2011; RADMAND et al., 2013).

Quanto à profilaxia antibiótica, ainda não existe diretriz padrão em pacientes candidatos a transplante hepático. Em pacientes com ascite, a profilaxia antibiótica parece ser necessária. O médico responsável deverá ser consultado para auxiliar nessa tomada de decisão (CRUZ PAMPLONA et al., 2011; PERDIGÃO et al., 2012; SILVA SANTOS et al., 2012; LEVENSON e KEENAN, 2013; RADMAND et al., 2013).

Devido à complexidade da condição geral dos pacientes com doença hepática crônica, o cirurgião-dentista deve estar atento a três aspectos essenciais:controle da hemostasia, controleda infecção e controle da dor (RADMAND et al., 2013). Assim, deve ser priorizado, sempre que possível, os procedimentos de alívio da dor e a abordagem dos quadros infecciosos. Em seguida, procedimentos restauradores e/ou provisórios devem ser realizados, devolvendo a função mastigatória e estética, habilitando o paciente ao transplante. Alguns procedimentos restauradores definitivos poderão ser postergados para a fase pós-transplante.

Durante o tratamento odontológico, na fase pré-transplante, o paciente deve ser orientado, monitorado e estimulado quanto aos cuidados com a saúde bucal. Nesta fase, é importante que o paciente incorpore e mantenha os cuidados com o controle da higiene bucal. Esses cuidados irão reduzir o risco de infecções de origem bucal na fase póstransplante, o que poderia acarretar na perda do órgão (SILVA SANTOS et al., 2012).

A realização de procedimentos minimamente invasivos, como procedimentos restauradores e protéticos, em indivíduos com hepatopatias, não está associada ao risco considerável de hemorragia. A dificuldade reside na avaliação do risco de sangramento em procedimentos com um moderado nível de invasão, como raspagem subgengival (SILVA SANTOS et al., 2012).

Ainda não existe consenso na literatura sobre o valor seguro de plaquetas ou RNI para a realização de procedimentos cirúrgicos. A experiência do Programa de Assistência Odontológica a Pacientes Transplantados da UFMG demonstra que contagem de plaquetas $\geq 50.000 / \mathrm{mm}^{3}$ e RNI $\leq 3,5$ são valores seguros para a realização de tais procedimentos. Esses parâmetros referenciais estão em conformidade com outros estudos (AFDHAL et al., 2008; SILVA SANTOS et al., 2012).

O uso de agente hemostático local em procedimentos cirúrgicos é de grande valor na obtenção de hemostasia pós-operatório imediato. Entretanto, esses agentes 
Atendimento odontológico a pacientes em fase de pré-transplante hepático: proposta de protocolo

hemostáticos locais podem aumentar o risco de infecção e atrasar a cicatrização (SILVA SANTOS et al., 2012). Portanto, deve-se analisar o sangramento durante o transoperatórioe, apenas caso seja excessivo, deve-se optar pelo uso desses agentes hemostáticos locais.

Além do uso de agentes hemostáticos locais, pode-se utilizar a terapia sistêmica com o objetivo de otimizar a hemostasia, essa terapia inclui infusões de produtos derivados do sangue (PFC, plaquetas, concentrado de hemácias, crioprecipitado, fator VIIa recombinante), ácido tranexâmico e injeções de vitamina K (AFDHAL et al., 2008; PERDIGÃO et al., 2012; RADMAND et al., 2013).A terapia sistêmica deve ser considerada apenas quando houver falha dos agentes hemostáticos locais. Para realizar essa terapêutica com segurança, deve-se analisar a condição sistêmica do paciente e interações medicamentosas.

Adicionalmente, é fundamental que o paciente, após a realização do transplante, retorne ao cirurgião-dentista para a realização de tratamentos definitivos e para manutenção preventiva, considerando que este fará uso de imunossupressor por um tempo prolongado.

A proposta esquematizada de protocolo para o atendimento de pacientes com hepatopatias em fase pré-transplante de fígado é apresentada na figura 1.

\section{CONSIDERAÇÕES FINAIS}

A assistência odontológica ao indivíduo candidato a transplante hepático pode ser realizada com total segurança pelo cirurgião dentista, desde que medidas simples, mas de extrema importância, sejam executadas. É fundamental a correta interpretação dos exames laboratoriais, assim como a prescrição de fármacos que promovam um menor dano ao sistema hepático. A partir desta constatação, graduandos participantes do projeto de extensão "Assistência Odontológica a Pacientes Transplantados de Fígado" não só atendem estes pacientes como também são capazes de elaborar e propor um protocolo de atenção básica embasados na literatura, articulando os pilares ensino, pesquisa e extensão.

Adicionalmente, o atendimento multidisciplinar garante uma assistência segura e de qualidade. A integração entre a odontologia e a equipe envolvida no processo de transplante gera melhores resultados para a saúde global do paciente tanto no período prétransplante quanto no período pós-transplante, e melhora a sua qualidade de vida. Esta 
Atendimento odontológico a pacientes em fase de pré-transplante hepático: proposta de protocolo

premissa é um exemplo da vivência da interdisciplinaridade e interprofissionalidade tão caras à extensão universitária.

O protocolo para atendimento de pacientes de transplante hepático proposto pelo projeto de extensão da Faculdade de Odontologia da UFMG “Assistência Odontológica a Pacientes Transplantados de Fígado”, disponibiliza à comunidade odontológica uma fonte atual e revisada sobre o atendimento do paciente que aguarda pelo transplante de fígado.Essa é uma expectativa deste projeto de extensão de cumprimento das diretrizes de impacto na sociedade e impacto na formação do estudante, possibilitando ao cirurgiãodentista o acesso a um conjunto de informações sobre as melhores estratégias e cuidados indispensáveis aos pacientes nesta fase. Dessa forma, oferece aos profissionais uma fonte com informações sistematizadas, com o objetivo de ajudá-los a se sentirem mais capacitados, seguros e aptos ao atendimento deste perfil de paciente.

\section{REFÊRENCIAS}

ÅBERG F, HELENIUS-HIETALA J, MEURMAN J, ISONIEMI H. Association between dental infections and the clinical course of chronic liver disease. Hepatology Research,v.44, n.3, 349-353, Apr. 2014.

AFDHAL, N.; MCHUTCHISON, J.; BROWN, R. Thrombocytopenia associated with chronic liver disease. J Hepatol, v.48, p.1000-1007, Jun. 2008.

CRUZ-PAMPLONA，M.; MARGAIX-MUÑOZ，M.; SARRIÓN-PÉREZ，M. Dental considerations in patients with liver disease. J Clin Exp Dent, v. 3, p.127-134, Feb.2011.

DI PROFIO, B.; INOUE, G.; MARUI, V.C.; FRANÇA, B.N.; ROMITO, G.A.; ORTEGA, K.L.; et al. Periodontal status of liver transplant candidates and healthy controls. J Periodontol, v.89, p.1383-1389, Dec. 2017.

FOX, R. When to Consider Liver Transplant During the Management of Chronic Liver Disease. Med Clin North Am,v.98, n.1, p. 153-168, Jan. 2014.

FREITAS, A. C.T; RAMPIM, A.T.; KUROGI, ANUNES, C.P.;COELHO, J.C.U. Impact of Meld Sodium on Liver Transplantation Waiting List. Arquivos de Gatroenterologia,v.32, n.3, p.e-1460, Dec. 2019.

GBD 2017 Cirrhosis Collaborators. The global, regional, and national burden of cirrhosis by causein 195 countries and territories, 1990-2017: a systematicanalysis for the Global Burden of Disease Study 2017. Lancet Gastroenterol Hepatol, v.5, n.3, p. 245-266, Mar.2020. 
Atendimento odontológico a pacientes em fase de pré-transplante hepático: proposta de protocolo

HELENIUS-HIETALA, J.; ÅBERG, F.; MEURMAN, J.; ISONIEMI, H. Increased infection risk postliver transplant without pretransplant dental treatment. Oral Diseases, v.19, n.3, p.271-278, July 2012.

KIMURA, Y.; SOSA, R.T.; SOTO-TRUJILLO, D.; SANDOVAL, Y.K.; CASIAN, C. Liver Transplant Complications RadiologistCan't Miss. Cureus, v. 12, n.6, p. e8465, June, 2020.

LEVENSON, M.S.; KEENAN, A.V. Dental Management of Liver Transplant Patient: Case Report. The New State Dental York Journal, v.79, p.16-18, Dec. 2013.

LINS, L.; AGUIAR, I.; CARVALHO, F.M.; SOUZA, L.; SARMENTO, V.; CODES, L.; BITTENCOURT, P.; PARANÁ, R.; BASTOS, J. Oral health and quality of life in candidates for liver transplantation. Transplantation Proceedings,v.49, n.4, p. 836-840, May 2017.

MEIRELLES JUNIOR, R. F.;SALVALAGGIO, P.; RESESNDE, M.B.; et al. Liver transplantation: history, outcomes and perspectives. Einstein (São Paulo), v. 13, n. 1, p. 149-152, Mar. 2015.

NATIONAL DATA. Acesso em: Setembro 18, 2020: https://optn.transplant.hrsa.gov/data/viewdata-reports/national-data/\#.

O’GRADY, J. Liver transplantation. Medicine, v.39, n.10, p.621-623, Oct. 2011.

PERDIGÃO, J.P.V.:; ALMEIDA, P.C.; ROCHA, T.D.S.; MOTA, M.R.L.; ALVES, A.P.N.N.; SOUSA, F.B.;SOARES, E.C.S.; SOUSA, F.B. Postoperativebleedingafter dental extraction in liverpretransplantpatients. Journal of Oral and MaxillSurgery, v.70, n.3, p.177-184, Mar. 2012.

RADMAND, R.; SCHILSKY, M.; JAKAB, S.; KHALAF, M.; FALACE DA. Pre-liver transplant protocols in dentistry. Oral Surg Oral Med Oral Pathol Oral Radiol, v.115, n.4, p.426-430, Apr. 2013.

SILVA SANTOS, P.S.; FERNANDES, K.S.; GALLOTTINI, M.H.C. Assessment and management of oral health in liver transplant candidates. Journal of Applied Oral Science, v. 20, n.2, p.241-245, Apr. 2012.

WARD, B.B.; WEIDEMAN, E.M. Long-Term Postoperative Bleeding After Dentoalveolar Surgery in the Pre transplant Liver Failure Patient. Journal of Oral and Maxillofacial Surgery, v.64, n.10, p.1469-1474, Oct. 2006.

ZAPATER, P.; LLANOS, L.; BARQUERO, C.; BELLOT, P.; PASCUAL, S.; CARNICER, F.; PALAZÓN, J.M.; GIMENEZ, P.; ESTEBAN, A.; LLORCA, L.; FRANCÉS, R.; HORGA, J.F.; SUCH, J. Acute Effects of Dipyrone on Renal Function in Patients with Cirrhosis: A Randomized Controlled Trial. Basic clinicalpharmacologytoxicology, v.116, n.3, p. 257-263, Mar. 2015.

Recebido em: 06/03/2020 
Atendimento odontológico a pacientes em fase de pré-transplante hepático: proposta de protocolo

Aceito em: 26/11/2020

FIGURA 1 - Proposta de protocolo para atendimento odontológico a pacientes hepatopatas

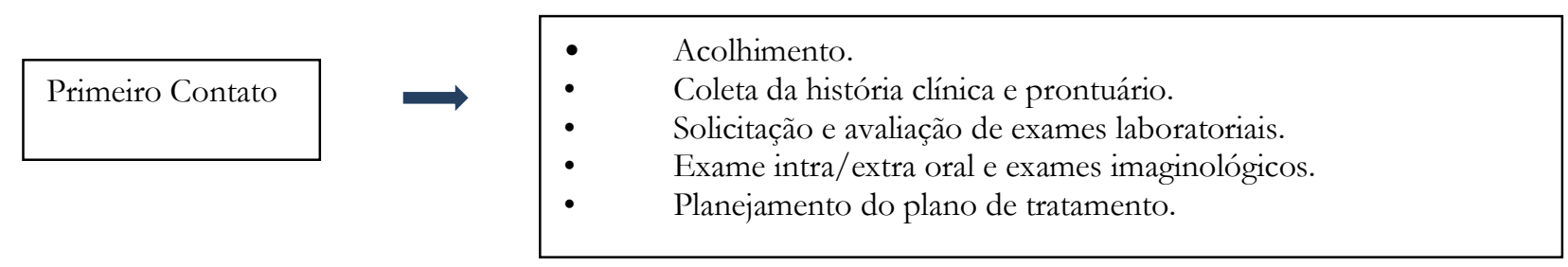


Atendimento odontológico a pacientes em fase de pré-transplante hepático: proposta de protocolo

\begin{tabular}{|l|l|}
\hline Início da Atenção & $\begin{array}{l}\text { Controle da dor e remoção de focos infecciosos. } \\
-\quad \text { Transfusão sanguínea em pacientes que apresentarem contagem } \\
\text { de plaquetas }<50.000 / \mathrm{mm}^{3} \text {. E valor limítrofe de RNI } 3,5 .\end{array}$ \\
$\begin{array}{l}\text { Procedimentos endodônticos e cirúrgicos requerem um cuidado } \\
\text { especial em relação àmedicação, devendo ter como analgésicos de } \\
\text { primeira escolha dipirona } 500 \mathrm{mg} \text { por até } 72 \text { horas ou paracetamol com } \\
\text { dosagem máxima de } 2 \mathrm{~g} / \text { dia. Não prescrever AINES. } \\
\text { Profilaxia antibiótica apenas em casos de Ascite ou hemorragia } \\
\text { gastrointestinal. } \\
\text { Orientação, monitoramento e estímulo quanto à saúde bucal. }\end{array}$ \\
\hline
\end{tabular}

Atenção básica para

liberação do paciente
Finalização da assistência e manutenção (após liberação médica)
- $\quad$ Procedimentos restauradores que devolvam ao paciente função e estética. Nesse estágio alguns tipos de tratamentos definitivos podem ser postergados para depois do transplante.

- Verificação da apreensão dos métodos de higienização oral e estímulo àcontinuação dos mesmos.

- Orientação, monitoramento e estímulo quanto à saúde bucal.

- Realização de tratamentos restauradores definitivos e manutenção preventiva.

- Manutenção da orientação, monitoramento e estímulo quanto à saúde bucal. 